\title{
Manifestations non verbales et stratégies d'écoute d'élèves de troisième cycle du primaire et stratégies d'intervention de leurs enseignants dans ce contexte
}

\author{
JOANNE PHARAND, LIZANNE LAFONTAINE \\ Université du Québec en Outaouais
}

\begin{abstract}
Résumé
La compréhension de l'oral implique la mise en pratique de stratégies d'écoute de la part des élèves et de stratégies d'intervention de la part des enseignants. À partir d'une recherche descriptive et exploratoire menée en 2009 auprès de 51 élèves de troisième cycle du primaire, nous avons répertorié un certain nombre de manifestations non verbales de l'écoute, de réactions au rappel à l'ordre des enseignants et des stratégies utilisées pour garder leur attention en classe. Pour leur part, les deux enseignants interrogés ont indiqué certaines stratégies employées pour recentrer les élèves à la tâche. Cet article présente les résultats de cette recherche.
\end{abstract}

\section{Problématique}

Aucune étude internationale ou nationale n'a encore mesuré la maîtrise de la compétence orale des élèves, dont l'écoute, en dépit du fait que les compétences à l'oral font partie intégrante de la littératie (Lafontaine, 2007; Lafontaine \& Messier, 2009). Par littératie, il faut entendre la «capacité d'utiliser le langage et les images, de formes riches et variées, pour lire, écrire, écouter, parler, voir, représenter et penser de façon critique » (ministère de 1'Éducation de 1'Ontario, 2006, p. 5). La littératie, volet oral, englobe les contextes de prise de parole, la production orale, le vocabulaire compris et utilisé, la compréhension d'un message, la capacité d'écoute, les interactions sociales dans des situations de communication scolaires ou extrascolaires ainsi que les liens étroits entre tous ces paramètres (Lafontaine, 2013). Dans le même ordre d'idées, l'oral, dont les stratégies d'écoute, favorise l'inclusion scolaire grâce à ses situations d'interactions entre les élèves qui permettent de faire naître un réseau social (Lafontaine, 2007; Dumais, 2010).

De plus, le Programme de formation de l'école québécoise (ministère de l'Éducation du Québec [MEQ], 2001), en français langue d'enseignement, propose la compétence disciplinaire Communiquer oralement. Dans cette compétence, on retrouve la composante « réagir aux propos entendus au cours d'une situation de communication orale » (p. 82). Dans le même esprit, l'un des critères d'évaluation de cette compétence se rapporte aux « réactions témoignant d'une écoute efficace » (p. 82). Dans ce programme, on fait également état de huit stratégies d'écoute dont quatre touchent les manifestations non verbales, soit «adopter une attitude d'ouverture; prendre une posture d'écoute (attention, regard dirigé); utiliser le langage non verbal pour montrer son incompréhension, son intérêt, son accord ou son désaccord; interpréter le langage non verbal (signes d'attention, d'intérêt ou d'ennui) » (p. 93). En 2011, le ministère de l'Éducation, du Loisir et du Sport (MELS) du Québec a publié un document portant sur la 
progression des apprentissages en oral dans lequel des manifestations observables des stratégies sont proposées afin d'orienter les enseignants dans leurs choix pédagogiques, à savoir :

- adopter une attitude d'ouverture : exprimer verbalement son accueil, recourir au langage non verbal (mimique, sourire, geste d'encouragement);

- prendre une posture d'écoute : se préparer à l'écoute (ex. : intention d'écoute, connaissances antérieures), recourir à des conduites qui favorisent la communication (ex. : attente, silence, geste), porter attention au langage non verbal des autres (ex. : regard dirigé), explorer des moyens pour organiser et retenir l'information;

- utiliser le langage non verbal pour démontrer son intérêt ou son désintérêt, son accord ou son désaccord, sa compréhension ou son incompréhension;

- interpréter le langage non verbal ${ }^{1}$ (MELS, 2011).

Même si ce document portant sur la progression des apprentissages en oral précise les manifestations à observer dans les stratégies du Programme de formation de l'école québécoise (MEQ, 2001), il reste qu'elles sont difficiles à traduire en classe et qu'elles demeurent très peu enseignées à l'heure actuelle (Nolin, 2012). En effet, plusieurs de ces manifestations observables de l'écoute ne permettent pas spécifiquement de savoir si l'élève écoute réellement; il faut s'en remettre à l'interprétation que l'enseignant peut faire d'une manifestation de l'écoute. Par exemple, diriger son regard dehors pourrait signifier que l'élève n'écoute pas alors qu'au contraire, il regarde à l'extérieur parce qu'il réfléchit. Également, Lafontaine (2007) a recensé plusieurs stratégies d'enseignement et d'apprentissage de l'écoute à travers des documents ministériels et des recherches, mais malgré les stratégies proposées, on ne sait pas comment l'élève s'y prend pour les opérationnaliser, particulièrement parce que celles-ci ne sont pas enseignées de manière explicite (Lafontaine, 2007; Lafontaine \& Messier, 2009; Nolin, 2012).

Cette recherche descriptive et exploratoire s'intéresse à l'exploration de stratégies d'écoute utilisées par des élèves lors de l'enseignement et de stratégies d'intervention utilisées par des enseignants dans ce contexte. Elle vise trois objectifs : 1) cibler les indices d'observation des manifestations non verbales de l'écoute chez les élèves; 2) identifier les stratégies utilisées par les élèves pour garder leur écoute lors de l'enseignement; 3) identifier les stratégies utilisées par les enseignants pour intervenir auprès de leurs élèves dans ce contexte.

\section{Cadre théorique}

Notre recherche s'inscrit dans le courant de l'oral médium et objet d'enseignement, à savoir que l'oral peut à la fois servir d'outil de transmission des connaissances, par exemple expliquer une notion oralement, et être un objet d'enseignement à part entière, par exemple l'enseignement explicite d'un objet de l'oral (volume de la voix, reformulation, argumentation) ou l'enseignement explicite d'une stratégie d'écoute et de prise de parole (comment utiliser son langage non verbal pour

${ }^{1}$ Le document sur la progression des apprentissages du MELS est en ligne et a été consulté le 16 janvier 2014 : http://www1.mels.gouv.qc.ca/progressionPrimaire/francaisEns/index.asp?page=conn com. 
démontrer son intérêt ou son désintérêt; prendre des risques de formulation pour exprimer son point de vue) (Dolz \& Schneuwly, 1998; Lafontaine \& Préfontaine, 2007; Lafontaine, 2013). Par le langage oral, l'élève développe et exprime sa pensée, il apprend, il effectue des transferts et reconstruit sa vision du monde. L'oral devient un outil de réflexion cognitive et métacognitive pour celui qui apprend et celui qui enseigne (Plessis-Bélair, 2004; Le Cunff, 2004).

Sur le plan de l'oral objet d'enseignement, la compréhension orale est le volet touché dans la présente recherche. La compréhension orale est un processus de réception active dans lequel un élève est amené à construire la signification d'un message, le remettre en contexte et établir des liens entre les informations contenues dans le message entendu et ses connaissances antérieures (Mordaunt \& Olson, 2010). Afin de mettre en œuvre sa compréhension orale, l'élève fait appel à des stratégies d'écoute qui sont des moyens ponctuels de répondre à une intention précise comme le cible notre recherche, à savoir prendre une posture d'écoute adéquate et une attitude d'ouverture à la réception et à la compréhension des messages (Lafontaine, 2007).

Pour Watzlawick, Helmick Beavin et Jackson (1972), la communication n'est pas uniquement composée de mots, de configurations et de sens, mais aussi de ses concomitants non verbaux et du langage du corps. Observer le va-et-vient entre les messages émis et reçus exige une observation attentive de ces échanges. Les manifestations non verbales de l'écoute font partie de cette observation. Pour répertorier ces manifestations, nous avons eu recours aux données d'une recherche antérieure portant sur les manifestations expressives des émotions dans la communication entre l'enseignant et les élèves lors de l'enseignement et montrant une écoute variable des élèves (Pharand, 2005). Ces manifestations expressives peuvent être observables. Comme le définit Christophe (1998), l'émotion est un processus à la fois individuel qui se manifeste lors d'un événement quelconque et multidimensionnel qui s'accompagne de manifestations physiologiques, expressives, cognitives et subjectives. Le langage émotionnel s'apparente très souvent au non-verbal (Pharand, 2013). Les manifestations non verbales observables comportent six expressions faciales universellement reconnues : la joie, la tristesse, la colère, la peur, la surprise et le dégoût auxquelles s'ajoutent, entre autres, l'intérêt et le désintérêt. Ces manifestations consistent également en des réponses corporelles musculaires telles que les postures et les gestes (Christophe, 1998).

Les postures font référence à des attitudes particulières du corps qui peuvent être convenables ou peu. Quant aux gestes, ils se rapportent au mouvement du corps (bras, mains, tête) volontaire ou involontaire, révélant un état psychologique ou visant à exprimer quelque chose. Pour les expressions faciales, il s'agit d'un ensemble de signes apparents, particulièrement sur le visage, par lequel se manifeste un état affectif (Pharand, 2005, 2013; Préfontaine, Lebrun \& Nachbauer, 1998). Les manifestations non verbales précitées ont servi d'indices d'observation pour la présente recherche et ont été classées selon trois caractéristiques : a) positives : manifestations favorables à l'écoute; b) neutres : peu ou pas d'effet sur l'écoute; c) négatives : défavorables à l'écoute des élèves (Pharand, 2005, 2013).

Le maintien d'une écoute attentive pendant l'enseignement fait appel à des stratégies variées de la part des élèves, mais aussi des enseignants. En quoi consiste une stratégie? Dans le monde de l'enseignement, une stratégie est une activité que l'enseignant ou l'élève développe pour surmonter certains obstacles (Bouchard, 2003). La 
personne utilise une ou des stratégies pour résoudre des problèmes. Il en est ainsi pour l'écoute: l'élève à qui on a enseigné l'écoute de manière explicite met en pratique différentes stratégies pour répondre à son intention d'écoute (Lafontaine, 2007; MÉO, 2008). Celui qui se retrouve dans une situation problématique doit croire qu'il est possible de s'ajuster pour s'approprier des stratégies (problem-focused) et pour planifier un changement (problem-solving). Tout cela dans un but d'enseignement approprié et d'apprentissages signifiants pour les élèves.

Pour Guérin (2008), une stratégie d'apprentissage est une catégorie d'actions métacognitives (capacité de jeter un certain regard sur son propre fonctionnement cognitif) ou cognitives utilisées dans une situation d'apprentissage donnée en vue de réaliser une tâche ou une activité scolaire. La taxonomie proposée par l'auteur est simple et comprend dix actions regroupées selon les stratégies métacognitives, soit anticiper et s'autoréguler; les stratégies cognitives de traitement de l'information, soit sélectionner, répéter, décomposer, élaborer et organiser; les stratégies cognitives d'exécution, soit évaluer, vérifier, reproduire et traduire. L'avantage de cette taxonomie est qu'elle s'applique dans la majorité des situations scolaires.

Pour Lafontaine et Pharand (2011), des stratégies d'écoute prédisposent également à l'apprentissage. Elles ont établi une correspondance entre ces stratégies et celles recensées dans les écrits. Globalement, les stratégies kinesthésiques coïncident au langage non verbal (prendre une posture d'écoute, interpréter le langage non verbal), les stratégies cognitives visent à répéter, reformuler, activer ses connaissances antérieures et les stratégies d'initiative ont pour but de vérifier sa compréhension du message. Si certaines stratégies métacognitives et cognitives de Guérin (2008) s'apparentent à celles de Lafontaine et Pharand (2011), les stratégies kinesthésiques ne sont pas abordées par Guérin (2008).

Berthiaume et Daele (2010) regroupent les stratégies d'enseignement sous cinq catégories : l'exposé magistral, le questionnement, la discussion, le travail individuel et le travail de groupe. Ces stratégies sont choisies en fonction du domaine et des objectifs pédagogiques poursuivis. Pour leur part, Laraoui, Morel et Leblanc (2014) rapportent d'autres stratégies : la pédagogie différenciée, l'enseignement par projet, la mémorisation, le modelage (enseignement stratégique). Bissonnette, Richard et Gauthier (2005) affirment l'efficacité de l'enseignement explicite dans les milieux défavorisés.

Bien que la présente recherche ne traite pas spécifiquement de stratégies d'enseignement comme telles, les stratégies d'intervention des enseignants peuvent correspondre à certaines de ces stratégies alors que les stratégies d'écoute des élèves peuvent être associées à certaines stratégies d'apprentissage leur permettant de maintenir leur attention en classe. La discussion à la suite de la présentation de nos résultats établira quelques liens avec ces stratégies.

\section{Méthodologie}

Nous expliquons en détail notre échantillon (types de recherche), le déroulement de notre recherche, nos instruments de collecte de données et notre méthode d'analyse des données. 


\section{Échantillon}

Cette recherche de types descriptif et exploratoire (Savoie-Zajc, \& Karsenti, 2000) a regroupé un échantillon intentionnel de deux enseignantes et de leurs élèves de $5^{\mathrm{e}}$ et $6^{\mathrm{e}}$ année du primaire (51 élèves; 26 garçons et 25 filles) d'une école de la commission scolaire des Draveurs, région de l'Outaouais au Québec. Une direction d'établissement, devenue chargée de cours à l'UQO, a servi d'intermédiaire pour approcher des enseignants de troisième cycle de son ancienne école. Sur une base volontaire, deux enseignants ayant plus de 10 ans d'expérience ont manifesté leur intérêt. Les critères de sélection des élèves ont reposé sur la volonté de chacun et le consentement signé des parents. Notons que l'enfant du parent qui a refusé de signer était absent lors de l'expérimentation. Notre choix d'élèves du troisième cycle du primaire s'explique par le fait qu'ils vivront, dans un avenir rapproché, une transition vers le secondaire où ils auront à écouter plusieurs disciplines enseignées par plusieurs personnes.

\section{Déroulement de l'expérimentation \\ L'expérimentation s'est déroulée en cinq étapes.}

1. D'abord, les enseignants ont présenté une activité d'enseignement d'une durée de 20 minutes en français (un enseignant) et en mathématiques (un enseignant) selon une formule pédagogique identique, soit un enseignement de type magistral avec la participation des élèves. Pendant l'enseignement, les élèves ont été filmés. La caméra a été déplacée trois fois pour prendre tous les élèves.

2. Par la suite, nous avons visionné l'activité d'enseignement deux fois dans chaque groupe pour que les élèves indiquent les manifestations non verbales observées chez eux suivant des fiches appropriées d'indices d'observation des postures, des gestes et des expressions faciales issue d'une recherche antérieure (Pharand, 2005). Précisons que les élèves observaient leurs propres manifestations, non pas celles de leurs camarades.

3. Après le visionnage, nous avons animé une entrevue de groupe semi-dirigée d'une durée de 20 minutes afin de cibler immédiatement les stratégies d'écoute des élèves et leurs réactions lorsque l'enseignant les rappelle à l'ordre.

4. Ensuite, chaque élève disposait d'une vingtaine de minutes pour remplir un questionnaire individuel afin de connaître leurs stratégies lors de situations moins favorables à leur écoute (situations issues d'une recherche antérieure, Pharand, 2005).

5. Après la période d'enseignement, nous avons fait l'entrevue semi-dirigée individuelle d'une durée de 15 minutes avec chaque enseignant pour identifier leurs stratégies d'intervention dans ce contexte. 


\section{Instruments de collecte de données}

Pour réaliser cette recherche, divers instruments de collecte de données ont été utilisés. Afin d'atteindre notre premier objectif, soit cibler les indices d'observation des manifestations non verbales de l'écoute chez les élèves, nous avons eu recours à trois fiches d'observation créées dans une recherche antérieure : une portant sur les postures, une autre sur les gestes et une dernière sur les expressions faciales des élèves. Les trois fiches sont présentées dans les tableaux 1-2-3. Rappelons que ces manifestations non verbales ont servi d'indices d'observation et précisons que les symboles $(+, \mathrm{n},-)$ signifiant positif, neutre et négatif ont reçu un niveau d'accord interjuge de $80 \%$ (Pharand, 2005, 2013). Ces fiches correspondent à la deuxième étape du déroulement.

\section{Tableau 1}

Fiche d'indices d'observation des postures des élèves

\begin{tabular}{|l|l|l|}
\hline & Postures à observer & Cochez \\
\hline $1 .+$ & S'asseoir droit, adossé à sa chaise & \\
2. + & S'asseoir de côté pour mieux voir le groupe ou l'enseignant & \\
3. + & Appuyer le torse sur le pupitre & \\
4. + & Croiser ses bras, appuyés sur le pupitre & \\
$5 .+$ & Tourner sa tête pour voir l'enseignant & \\
6. + & Se pencher pour écrire ou lire & \\
7. $\mathrm{n}$ & Se retourner derrière pour regarder un élève & \\
8. $\mathrm{n}$ & Soutenir sa tête avec une main & \\
9. $\mathrm{n}$ & S'étirer les bras & \\
10. $\mathrm{n}$ & Se balancer sur sa chaise & \\
11. $\mathrm{n}$ & Appuyer ses pieds sur la base du pupitre & \\
12. $\mathrm{n}$ & Se lever, se déplacer & \\
13. - & Coucher sa tête sur un bras ou les bras / sur le pupitre & \\
14. - & Glisser les fesses sur sa chaise & \\
15. - & Placer le haut de son corps sur le pupitre & \\
16. - & Appuyer les genoux sur le pupitre & \\
17. - & S'asseoir sur ses talons / sa jambe & \\
18. & Autres & \\
\hline
\end{tabular}


Tableau 2

Fiche d'indices d'observation des gestes des élèves

\begin{tabular}{|c|c|c|}
\hline & Gestes à observer & Cochez \\
\hline $1 .+$ & Lever le bras pour répondre aux questions & \\
\hline 2. + & Se servir de son taille-crayon & \\
\hline 3. + & Pointer une affiche ou un cahier & \\
\hline 4. + & Chercher dans son étui à crayons/ les livres & \\
\hline 5. + & Écrire, effacer & \\
\hline 6. $\mathrm{n}$ & Laisser tomber le bras levé derrière soi & \\
\hline 7. $\mathrm{n}$ & Tenir le bras levé avec l'autre bras / la manche de chandail & \\
\hline 8. $\mathrm{n}$ & Agiter la main pour attirer l'attention & \\
\hline 9. $\mathrm{n}$ & Se gratter la main/le cou/le nez/l'œil/la tête/le front/l'oreille & \\
\hline 10. - & Porter à sa bouche ses doigts/crayon/règle & \\
\hline 11. - & Jouer avec des objets devant soi & \\
\hline 12. - & $\begin{array}{l}\text { Jouer avec les parties du corps : doigts/nez/bouche/cheveux } \\
\text { /menton/ ongles }\end{array}$ & \\
\hline 13. - & Balancer ses pieds & \\
\hline 14. - & Se frapper la joue/la tête avec son crayon & \\
\hline 15. - & Regarder dehors/un objet/sur la feuille du voisin & \\
\hline 16. - & Dessiner pendant la leçon & \\
\hline 17. - & Bailler & \\
\hline 18. - & Se tirailler avec son voisin & \\
\hline 19. & Autres & \\
\hline
\end{tabular}

\section{Tableau 3}

Fiche d'indices d'observation des expressions faciales des élèves

\begin{tabular}{|l|l|l|}
\hline & Expressions faciales observées & Cochez \\
\hline $1 .+$ & Intérêt & \\
$2 .+$ & Joie & \\
$3 .+$ & Surprise & \\
$4 .+$ & Autres & \\
\hline $1 .-$ & Désintérêt (fatigue, lassitude, ennui) & \\
2. - & Anxiété (incertitude) & \\
3. - & Joie (énervement) & \\
4. - & Autres & \\
\hline
\end{tabular}

Afin de bien repérer ces indices, il est important de noter que les élèves ont reçu, au préalable, une définition opérationnelle des manifestations (indices) : postures, gestes et expressions faciales. De plus, ces définitions étaient indiquées au début des fiches. Un premier visionnage a permis de cibler les postures observées. Un second visionnage a 
permis de cocher les gestes et les expressions faciales. Cette activité a duré environ 40 minutes.

En ce qui concerne notre deuxième objectif, c'est-à-dire identifier les stratégies utilisées par les élèves pour garder leur écoute lors de l'enseignement, nous avons utilisé deux instruments. Premièrement, nous avons réalisé une entrevue de groupe à la suite du visionnage de l'enseignement. Le schéma d'entrevue comprend quatre questions indiquées à l'encadré 1 pour connaitre leurs stratégies, mais aussi leurs réactions quant au rappel à l'ordre de l'enseignant. Cette entrevue de groupe correspond à la troisième étape du déroulement.

1. Quand vous adoptez certaines postures, quelles stratégies utilisez-vous pour garder votre attention et écouter lors de l'enseignement?

2. Quand votre enseignante vous interpelle par rapport à votre posture, que faites-vous? Est-ce que cela vous permet de mieux écouter? Si oui pourquoi?

3. Quand vous posez des gestes qui distraient votre attention pendant l'enseignement, quels moyens utilisez-vous pour continuer à écouter la leçon?

4. Quand votre enseignante intervient sur les gestes que vous posez pendant la leçon, que faites-vous pour garder votre attention? Est-ce que son intervention vous permet de mieux écouter?

Encadré 1. Schéma d'entrevue de groupe pour les élèves

Deuxièmement, les élèves ont rempli individuellement un questionnaire portant sur leurs stratégies d'écoute en lien avec des situations moins favorables à l'écoute. Le questionnaire compte 19 situations exposées dans l'encadré 2 et correspond à la quatrième étape du déroulement.

Consigne de départ : si tu as vécu ou si tu vis des situations semblables, écris les stratégies que tu utilises pour garder ton attention et écouter pendant l'enseignement. Si tu ne te sens pas concerné, passe à l'autre situation.

\section{Quelles stratégies utilises-tu pour garder ton attention et écouter quand :}

1. l'enseignante répète ce que tu sais déjà?

2. tu trouves les explications trop longues?

3. tu juges la matière trop facile?

4. tu trouves la matière difficile?

5. tu ne comprends pas les explications?

6. les autres font des bruits dérangeants?

7. les autres n'arrêtent pas de bavarder?

8. tu t'ennuies?

9. tu es fatigué?

10. tu es démotivé?

11. tu es préoccupé par des choses que tu juges plus importantes que l'école?

12. l'enseignante est stressée et de mauvaise humeur? 
13. tu as vécu une chicane avec des amis?

14. tu crains d'être rejeté par un(e) ami (e)?

15. tu vis des problèmes familiaux?

16. les autres refusent de t'aider à comprendre?

17. tu te trouves complètement perdu et que tu ne comprends pas les explications?

18 tu te laisses influencer par les niaiseries de certains élèves?

19. ton désir de faire rire les autres prend toute la place?

Encadré 2. Questionnaire individuel aux élèves

Ces questions résultent d'une analyse d'entrevues semi-dirigées menées auprès de 129 élèves des deuxième et troisième cycles du primaire de la région de l'Outaouais sur les motifs évoqués relativement aux émotions jugées défavorables à l'écoute en classe (Pharand, 2005, 2013).

Finalement, pour atteindre notre troisième objectif, soit identifier les stratégies utilisées par les enseignants pour intervenir auprès de leurs élèves dans ce contexte, nous avons réalisé une entrevue individuelle avec chacun d'eux. Le schéma d'entrevue présenté dans l'encadré 3 comprend trois questions portant sur les postures, les gestes et les émotions qu'ont pu observer les enseignants par rapport aux élèves. Cette entrevue correspond à la dernière étape du déroulement. Alors qu'une chercheuse surveillait la passation du questionnaire auprès des élèves, l'autre chercheuse procédait à l'entrevue auprès des enseignants.

1. Quand vous observez certaines postures chez vos élèves lorsque vous enseignez, quelles interventions faites-vous?

2. Quand vous observez certains gestes dérangeants chez vos élèves lorsque vous enseignez, que faites-vous?

3. Quand vous constatez que des élèves peuvent vivre des émotions pouvant nuire à leur écoute pendant l'enseignement, comment faites-vous pour recentrer leur attention?

(exemples : désintérêt, anxiété, joie excessive, autre,...)

Encadré 3. Schéma d'entrevue individuelle pour les enseignants

Rappelons enfin que les trois tableaux et l'encadré 2, comme instruments de collecte de données, ont été validés (contrôle de concordance par fidélité interjuge) dans une recherche antérieure (Pharand, 2005). Les encadrés 1 et 3 sont propres à la présente recherche.

\section{Méthode d'analyse des données}

Les données ont été traitées et codées en fonction des objectifs de la recherche aux moyens des instruments présentés précédemment. Les manifestations non verbales d'écoute ont été observées à partir du tournage vidéo de chaque groupe et des fiches d'observation appropriées. Pour l'entrevue de groupe, un code a été attribué à chaque classe et à chaque élève suivant la prise de parole et le sexe. Par exemple, la deuxième 
fille de la classe de l'enseignant E1 à prendre la parole a reçu le code E1-F12. Ainsi, le contexte initial des unités de sens est rapidement accessible afin d'assurer la validité des données classées selon les stratégies émergentes et identifiées. Des extraits d'entrevue permettent de qualifier les stratégies évoquées. Enfin, pour le questionnaire individuel, un code semblable à celui des entrevues a été utilisé. Le sexe des participants est indiqué afin de tenir compte de cette variable dans l'analyse.

L'analyse qualitative de contenu (Bardin, 2007) a été complétée par le comptage des occurrences de chacune des manifestations non verbales: on peut parler ici d'une analyse quantitative et de l'utilisation d'une méthodologie mixte. Elle regroupe les indices d'observation (manifestations non verbales qui ont été quantifiées dans les fiches d'indices d'observation) ainsi que les stratégies dominantes de l'écoute des élèves et les stratégies d'intervention des enseignants pendant l'enseignement (analyse qualitative des entrevues et du questionnaire). Précisons également qu'à la suite de l'analyse des entrevues auprès des participants et du questionnaire individuel auprès des élèves, nos données ont subi un traitement duquel a émergé une catégorisation des stratégies d'écoute des élèves et des stratégies d'intervention des enseignants.

\section{Résultats et analyse}

Les résultats sont présentés en fonction des trois objectifs de la recherche : 1) cibler les indices d'observation des manifestations non verbales de l'écoute chez les élèves; 2) identifier les stratégies utilisées par les élèves pour garder leur écoute lors de l'enseignement; 3) identifier les stratégies utilisées par les enseignantes pour intervenir auprès de leurs élèves dans ce contexte.

Rappelons que le déroulement de l'expérimentation s'est effectué en cinq étapes. La première étape consistait à filmer les élèves lors d'une activité d'enseignement. La deuxième visait à noter les manifestations non verbales observées par les élèves à partir des fiches d'indices d'observation (tableaux 1-2-3) appropriés (objectif 1). Les étapes trois et quatre concernent deux instruments : l'entrevue de groupe (encadré 1) afin de recueillir certaines stratégies d'écoute des élèves en lien avec leurs postures et leurs gestes et quelques réactions lorsqu'ils sont ramenés à l'ordre par leur enseignant; puis le questionnaire individuel (encadré 2) pour connaitre les stratégies d'écoute des élèves lors de situations particulières (objectif 2). L'étape cinq rapporte, au moyen de l'entrevue individuelle (encadré 3), les stratégies d'intervention émises par les enseignants (objectif 3 ). Des extraits d'entrevues ou de réponses au questionnaire viennent ponctuer ces résultats.

Indices d'observation des manifestations non verbales des élèves

De prime abord, il est important de noter que les indices d'observation (postures, gestes et expressions faciales) jugés positifs $(+)$ indiquent une bonne écoute, ceux notés neutres (n) ont peu ou pas d'effet sur l'écoute et ceux jugés négatifs (-) signifient une moins bonne écoute de la part des élèves pendant l'enseignement (Pharand, 2005). Le tableau 4 montre les postures identifiées par les 51 élèves, soit 25 filles et 26 garçons, et le calcul des occurrences. Si les occurrences dépassent le nombre d'élèves, c'est que certains d'entre eux ont noté deux fois la posture observée durant le visionnage de la vidéo. 
Tableau 4

Postures des élèves par ordre d'importance

\begin{tabular}{|c|c|}
\hline Les filles & Les garçons \\
\hline $\begin{array}{l}\text { 1. Soutenir sa tête avec une main }(\mathrm{n}), \\
\text { 25,30 } \\
\text { 2. Tourner sa tête pour voir l'enseignant } \\
(+), 19,10 \\
\text { 3. Se pencher pour écrire ou lire }(+), 15,10 \\
\text { 4. Croiser ses bras, appuyés sur le pupitre } \\
(+), 14,20 \\
\text { 5. S'asseoir de côté pour mieux voir le } \\
\text { groupe }(+), 14,10 \\
\text { 6. Se retourner derrière pour regarder un } \\
\text { élève }(\mathrm{n}), 10,30 \\
\text { 7. S'asseoir droit, adossé à sa chaise }(+), \\
\text { 10,20 }\end{array}$ & $\begin{array}{l}\text { 1. Soutenir sa tête avec une main }(\mathrm{n}), \\
29,10 \\
\text { 2. Se retourner derrière pour regarder un } \\
\text { élève }(\mathrm{n}), 16.10 \\
\text { 3. Appuyer le torse sur le pupitre }(+), 15 \\
\text { 4. Se pencher pour écrire ou lire }(+), 12,30 \\
\text { 5. Croiser ses bras, appuyés sur le pupitre } \\
(+), 12,10 \\
\text { 6. Tourner sa tête pour voir l'enseignant } \\
\text { (+), } 10,30 \\
\text { 7. Appuyer ses pieds sur la base du pupitre } \\
\text { (n), } 9,20\end{array}$ \\
\hline
\end{tabular}

Soutenir sa tête avec la main obtient le premier rang chez les deux sexes. La tête semble avoir besoin d'un appui physique pour maintenir l'écoute : Si je m'accote sur mon coude, ça m'aide à penser pour comprendre (E1-G5). L'ordre d'importance attribué par les filles diffère quelque peu de celui des garçons. Il est intéressant de noter que les filles adoptent davantage une posture droite, alors que les garçons s'appuient davantage sur leur bureau ou mettent leurs pieds sur la base du pupitre. Somme toute, plusieurs postures indiquent une bonne écoute des élèves. Le tableau 5 illustre les gestes identifiés par les élèves et le calcul des occurrences.

\section{Tableau 5}

Gestes des élèves par ordre d'importance

\begin{tabular}{|c|c|}
\hline Les filles & Les garçons \\
\hline 1. Se gratter la main/le cou/l'œil/la tête/ & 1. Se gratter la main/le cou/l'œil/la tête/le \\
\hline $\begin{array}{l}\text { 2. Jouer avec les parties du corps : doigts/ } \\
\text { nez/bouche/cheveux/menton/ongles }(-), 20,2\end{array}$ & $\begin{array}{l}\text { 2. Jouer avec les parties du corps : doigts/ } \\
\text { nez/bouche/cheveux/menton/ongles }(-), 20\end{array}$ \\
\hline 3. Bailler (-), 15,50 & 3. Bailler $(-), 18,20$ \\
\hline 4. Écrire, effacer $(+), 11,10$ & 4. Lever le bras pour répondre aux \\
\hline 5. Lever le bras pour répondre aux questions & questions $(+), 18,20$ \\
\hline$(+), 11$ & 5. Écrire, effacer $(+), 17$ \\
\hline $\begin{array}{l}\text { 6. Regarder dehors/un objet/sur la feuille } \\
\text { du voisin }(-), 10,40\end{array}$ & 6. Jouer avec des objets devant soi (-), 13 \\
\hline
\end{tabular}

Pendant l'enseignement plusieurs gestes se font, même involontairement. Pour la majorité des élèves se gratter demeure un geste fréquent qui ne nuit pas à l'écoute. Jouer 
avec les parties du corps est noté de manière négative à l'écoute. Il convient cependant de préciser que ces gestes en soi ne dérangent pas, mais que c'est plutôt le jeu qui déconcentre. Quant au bâillement, il peut signifier l'ennui ou la fatigue qui finissent par affecter l'écoute. Pour tous les élèves, les gestes de concentration à la tâche sont jugées positifs. Les gestes montrent également des habitudes qui peuvent sembler anodines, mais qui, à la longue, peuvent déconcentrer. Jouer avec des objets pour garder sa concentration est plus fréquent chez les garçons. Le tableau 6 présente les expressions faciales observées par les élèves et le calcul des occurrences.

Tableau 6

Expressions faciales des élèves par ordre d'importance

\begin{tabular}{|l|l|}
\hline Les filles & Les garçons \\
\hline 1. Désintérêt (et fatigue) (-), 19 & 1. Désintérêt (et fatigue) (-), 19,6 \\
2. Intérêt $(+), 6,20$ & 2. Intérêt $(+), 10$ \\
3. Joie $(+), 5$ & 3. Surprise $(+), 8,4$ \\
\hline
\end{tabular}

Les expressions faciales dévoilent un désintérêt lors de l'enseignement. Cela peut être attribuable à l'état d'esprit des élèves (fatigue, ennui) au moment du tournage, au type d'activité plutôt magistrale (révision de l'accord du participe passé dans un groupe et des exposants en mathématiques dans l'autre) ou au fait que sur les 20 minutes de visionnage, les élèves voient leur intérêt diminuer à certains moments. À contrario, les expressions d'intérêt, de joie et de surprise découlent de stratégies stimulantes pour présenter la matière et faire participer les élèves.

Entrevue de groupe et réactions aux interventions des enseignants

La transcription intégrale des deux entrevues de groupe menées après le visionnage devant les deux classes révèle que les postures vont de pair avec l'intérêt des élèves, leur humeur et leur compréhension de la matière. Le corps se place droit quand l'intérêt est évident et il s'affaisse quand le désintérêt s'installe.

Ça dépend en quelque sorte du cours. Je vais adopter une posture bien étendue parce que je n'aime pas bien ça. Si c'est un sujet assez intéressant, bien là, j'vais avoir une meilleure posture. E1-G1

Quand je comprends, je me mets vraiment droit, mais si je ne comprends pas, je suis dans la lune, alors... j'accote mon menton sur le bureau. F1-G4

Lorsque l'enseignant intervient auprès d'eux, des élèves disent écouter et trouver ce rappel à l'ordre efficace. D'autres le jugent inefficace, car ils finissent par reprendre leur posture jugée négative (ex. : coucher sa tête sur le pupitre). Cependant, tous les élèves conviennent que ce rappel est, malgré tout, nécessaire au recadrage de leur attention. 
Quand le professeur te rappelle, c'est souvent parce que t'es dans la lune ou que t'écoutes pas... ça te permet de mieux t'asseoir puis de pouvoir mieux écouter. E1-G7

Ça me rappelle de me réveiller puis de me mettre droit. Ça marche pendant cinq minutes... après... elle me le redit, ça marche cinq minutes... pendant une heure... Ça me permet de revenir dans l'enseignement au moins. E1-G8

Concernant les gestes posés pendant l'enseignement, les entrevues de groupe indiquent que le bruit causé par la manipulation d'objets semble déranger davantage que les objets que les élèves ont dans les mains.

Moi, quand je prends un petit objet, je deviens comme dans la lune, fait que je le fais bouger puis quand il tombe, ça me fait sauter... et je redeviens concentrée. E1-G13

J'vais souvent être distraite par les bruits... j'vais essayer de me distraire avec quelque chose qui ne fait pas de bruit. E1-F5

Quant aux rappels de l'enseignant, des élèves (notamment les garçons) disent éprouver le besoin d'avoir quelque chose dans les mains pour écouter et avoir de la difficulté à résister au fait de ne rien prendre. L'intervention de l'enseignant aide au début, mais ne semble pas durable.

J'vais le ranger, mais j'vais prendre un autre objet. E2-G18

Je fais me calmer pour cinq minutes, puis je vais recommencer à être dans la lune.... C'est une habitude! E1-G14

Pour connaitre les stratégies d'écoute des élèves par rapport à leurs expressions faciales, nous avons opté pour un questionnaire individuel relatant diverses situations où l'intérêt des apprenants est mis à l'épreuve lors de l'enseignement (Pharand, 2005). Le point suivant rapporte ces résultats.

Questionnaire individuel et stratégies d'écoute des élèves

L'analyse des réponses obtenues par le questionnaire individuel a mené au regroupement des stratégies utilisées par les élèves en trois catégories: stratégies kinesthésiques, cognitives et d'initiative (Lafontaine et Pharand, 2011). Le tableau 7 les présente. 
Tableau 7

Stratégies d'écoute des élèves issues du questionnaire

\author{
Stratégies kinesthésiques/Pour garder mon attention, je : \\ Joue avec un objet pendant que l'enseignant parle. \\ Dessine pendant que l'enseignant parle. \\ M'assois droit, prends une posture confortable. \\ Me couche sur mon bureau. \\ M'appuie sur mon bras, ma main, mes coudes, ma joue, mon menton. \\ Regarde le tableau. \\ Regarde autour de moi, regarde par la fenêtre. \\ Me secoue pour me réveiller. \\ Me couche plus tôt la veille. \\ Baille pour chasser la fatigue.
}

\title{
Stratégies cognitives/Pour garder mon attention, je :
}

Me chante une chanson en lien avec la matière ou chante les explications de

l'enseignant.

Me parle pour me dire d'écouter, pour m'encourager à écouter, me motiver, pour devenir meilleur.

Pense à ce que la notion m'apportera dans mon futur métier, dans l'avenir, au métier que je ferai plus tard.

Me fais un court résumé dans ma tête ou sur papier de tout ce que l'enseignant dit, me répète trois fois ce que l'enseignant a dit.

Prends des notes.

Me calme.

Me concentre pour écouter au cas où il y aurait des nouvelles notions ou d'autres explications, même si je connais déjà la matière, me concentrer pour bien réviser, pour ceux qui ne connaissent pas la matière, pour vérifier si j'ai oublié quelque chose.

Me concentre pour mieux comprendre.

Me concentre sur l'enseignant (sa voix, son regard, etc.).

Oublie les chicanes avec les amis ou les problèmes familiaux pour me concentrer sur la matière, règle le problème à la récréation ou à la maison.

Stratégies d'initiative/Pour garder mon attention, je :

Pose des questions à l'enseignant, à mes camarades, à mes parents.

Demande l'aide à un ami, à l'enseignant, aux parents.

Réponds aux questions de l'enseignant.

Vais voir l'enseignant après les explications, lui demande de répéter les explications.

Demande aux autres de ne plus parler, de ne pas déranger ou les ignore.

Cet ensemble de stratégies témoignent des efforts déployés par les élèves pour écouter, mais aussi pour comprendre, pour mémoriser et pour apprendre. Ils font preuve d'ingéniosité, de créativité et de bonnes intentions. Ils tentent en quelque sorte de répondre aux exigences de l'école, soit d'écouter l'enseignant lorsqu'il enseigne. Que signifient ces stratégies? 
Stratégies kinesthésiques. Les stratégies kinesthésiques démontrent ce que font les élèves avec leur corps pour manifester leur écoute. Il est intéressant de constater que, pour les élèves, jouer avec un objet ou dessiner pendant que l'enseignant parle, se coucher sur son bureau, s'appuyer, regarder autour de soi ou par la fenêtre, bailler pour chasser la fatigue sont des manifestations non verbales d'écoute et non pas nécessairement de désintérêt. Ils trouvent des stratégies qui se veulent efficaces notamment quand ils connaissent ou non la matière, que les explications de l'enseignant sont longues, qu'ils s'ennuient ou qu'ils sont fatigués.

\section{Je dessine ce qu'elle dit sous forme de bande dessinée. E1-G8}

Il faut que j'aie quelque chose dans les mains, sinon je vais être dans la lune. E1-F9

Stratégies cognitives. Les stratégies cognitives font état de l'activité mentale des élèves. Ils évoquent des stratégies assez originales comme : se chanter une chanson en lien avec la matière expliquée par l'enseignant; se faire un résumé dans sa tête de ce que dit l'enseignant; oublier les chicanes personnelles pour mieux se concentrer. À cela s'ajoutent des stratégies plus usuelles comme se parler à soi-même, se concentrer sur l'enseignant, prendre des notes. Somme toute, ces stratégies sont plutôt neutres sur le plan physique, et il est certes difficile de savoir à quel moment les élèves les utilisent pendant l'écoute. Néanmoins, elles semblent efficaces pour certains élèves.

Je vais réciter ses paroles dans ma tête ou sur papier. E2-F22

Je me concentre le plus possible et je fais tourner mes petites cellules grises. E1-F6

\section{J'essaie de cacher mes émotions face à cette chose à l'école. E2-F17}

Stratégies d'initiative. Les stratégies d'initiative sont celles que les élèves mettent de l'avant pour écouter. Manifester son écoute se fait en posant et en répondant à des questions, en demandant de l'aide à l'enseignant ou à ses pairs et en demandant à autrui de ne pas déranger, bref, en prenant diverses initiatives pour communiquer verbalement son besoin. Pour le bruit et le bavardage, la majorité des répondants affirme demander à l'autre de se taire, même si cela est parfois pénible, car ils ne veulent déplaire à leurs amis ou trouvent parfois le bavardage intéressant. Ces stratégies montrent le besoin des élèves de comprendre la matière pour avancer et réussir sans être continuellement dérangés.

Je pose des questions jusqu'à en être satisfait. E2-G24

Si les autres font du bruit, je fais comme si j'étais dans une grosse bulle et que je pouvais entendre seulement le professeur. C'est pratique, essayez! E2F19 
Toutes les stratégies d'écoute utilisées par les élèves trouvent écho dans celles recensées dans les écrits. Une correspondance intéressante est présentée dans Lafontaine et Pharand (2011).

\section{Entrevue individuelle et stratégies d'intervention des enseignants}

L'analyse des réponses à l'entrevue individuelle des enseignants présente les stratégies d'intervention qu'ils disent utiliser par rapport aux postures, aux gestes observés et aux expressions faciales d'émotions perçues et vécues par les élèves. Cette analyse a mené au regroupement des stratégies d'intervention évoquées en trois catégories: stratégies kinesthésiques, cognitives et affectives. Même s'il s'agit des réponses de deux enseignants seulement, nous estimons que leurs idées peuvent rejoindre celles d'autres enseignants. Le tableau 8 présente l'ensemble de ces stratégies.

\section{Tableau 8}

Stratégies d'interventions des enseignants issues de l'entrevue

\begin{tabular}{|c|c|c|}
\hline Stratégies kinesthésiques & Stratégies cognitives & Stratégies affectives \\
\hline $\begin{array}{l}\text { Par rapport aux postures } \\
\text { - Demander par signes. } \\
\text { - Demander verbalement de } \\
\text { s'asseoir correctement (si } \\
\text { répétitif). } \\
\text {-Passer à côté, replacer } \\
\text { discrètement l'élève. } \\
\text { Par rapport aux gestes } \\
\text { - Faire des signes. } \\
\text { - Passer à côté, enlever } \\
\text { l'objet de la main, mettre } \\
\text { sur son bureau. } \\
\text { - Placer l'élève à l'arrière } \\
\text { de la classe. } \\
\text { Par rapport aux émotions } \\
\text { Colère } \\
\text { - L'inviter dans le corridor } \\
\text { pour qu'il s'assoie. } \\
\text { - L'inviter à aller aux } \\
\text { toilettes pour s'asperger } \\
\text { d'eau, se rafraîchir. }\end{array}$ & $\begin{array}{l}\text { Par rapport aux gestes } \\
\text { - Dire verbalement d'arrêter } \\
\text { devant la classe. } \\
\text { - Doser les interventions } \\
\text { (élèves kinesthésiques). } \\
\text { - Établir un système de } \\
\text { discipline avec règlements. } \\
\text { - Leur demander d'indiquer } \\
\text { des rappels dans l'agenda. } \\
\text { - S'assurer que l'élève } \\
\text { regarde l'enseignant. } \\
\text { - S'assurer que l'élève est } \\
\text { attentif à la tâche. } \\
\text { - Faire répéter l'élève qui } \\
\text { ne comprend pas la tâche. } \\
\text { Par rapport aux émotions } \\
\text { Joie (surexcitation) } \\
\text { - Le ramener à la tâche, le } \\
\text { remettre en position pour } \\
\text { que lui se questionne. } \\
\text { Désintérêt } \\
\text { - Le ramener à la tâche en } \\
\text { le raisonnant. } \\
\text { Colère } \\
\text { - S'adresser à la personne } \\
\text { pour essayer de calmer la } \\
\text { situation et entrevoir des }\end{array}$ & $\begin{array}{l}\text { Par rapport aux émotions } \\
\text { Tristesse } \\
\text { - Voir l'élève directement. } \\
\text { - Lui parler juste à lui. } \\
\text { Joie (surexcitation) } \\
\text { - Demander à l'élève de se } \\
\text { calmer, de se repositionner. } \\
\text { - Intervenir parfois en } \\
\text { groupe, parfois en } \\
\text { individuel. } \\
\text { - Intervenir avec humour. } \\
\text { - Faire comprendre qu'il y a } \\
\text { un temps pour rire, puis un } \\
\text { temps pour revenir au } \\
\text { sérieux... se reprendre en } \\
\text { main. } \\
\text { - Doser et s'assurer que } \\
\text { l'ensemble des élèves est } \\
\text { capable de suivre. } \\
\text { - Changer son scénario, } \\
\text { s'adapter, s'ajuster. } \\
\text { Désintérêt } \\
\text { - Dire à haute voix à } \\
\text { l'élève. } \\
\text { - Sortir de la lune } \\
\text { verbalement ou en } \\
\text { chuchotant à l'élève. } \\
\text { Colère }\end{array}$ \\
\hline
\end{tabular}




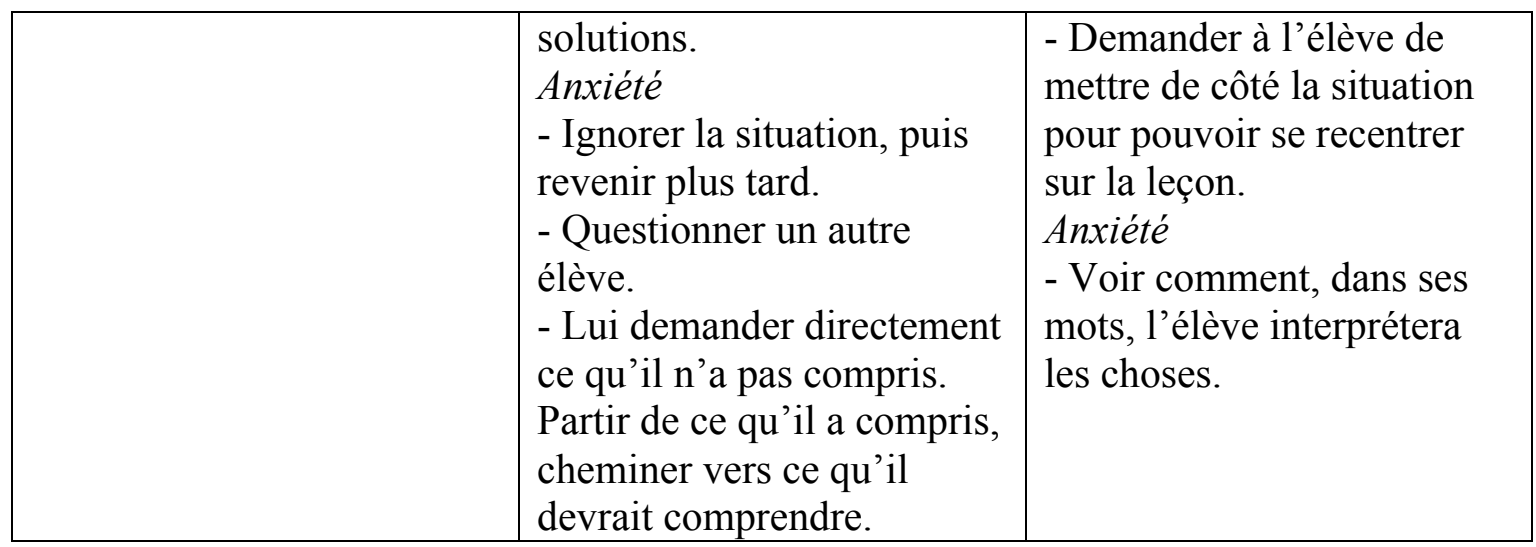

Cet ensemble de stratégies confirme la nécessité pour les enseignants d'intervenir auprès des élèves afin de passer la matière au programme et de s'assurer que tous les apprenants sont présents physiquement et mentalement. Que signifient ces stratégies?

Stratégies kinesthésiques. Les stratégies kinesthésiques montrent ce que font ou disent les enseignants pour ramener les élèves à l'ordre. Les demandes par signes ou verbalement sont utilisées. Le déplacement vers un élève distrait pour le replacer ou lui enlever un objet est une autre stratégie. Un élève peut aussi être changé de place. Enfin, si un élève manifeste de la colère, il peut être invité à s'assoir dans le corridor ou à aller au lavabo pour s'asperger d'eau, se rafraîchir.

Les élèves se balancent sur les deux pattes de chaise. Je fais juste leur demander ou je leur fais signe avec mes doigts, quatre pattes. E1

Stratégies cognitives. Les stratégies cognitives font état de l'activation mentale demandée aux élèves pour retrouver leur attention. Les enseignants utilisent la parole et le système de gestion de la classe pour rappeler les règlements tout en dosant les interventions auprès des élèves kinesthésiques et en faisant répéter l'élève qui ne comprend pas. Le regard dirigé vers l'enseignant demeure une exigence annoncée. Des stratégies cognitives sont utilisées pour raisonner les élèves par rapport à diverses manifestations émotionnelles observées. On souhaite que les élèves s'autorégulent dans leurs manifestations, qu'ils reviennent à la tâche par eux-mêmes. Pour certaines émotions, comme l'anxiété, le soutien des enseignants est présent et leurs stratégies sont variées.

Il y a des élèves dans la classe qui sont très kinesthésiques... il faut les laisser jouer avec leurs affaires, leurs mains ou n'importe quoi pour que leur attention soit là. E1

Je m'assure que les yeux sont sur moi... si l'élève ne comprend pas la tâche, je la fais répéter par un autre élève. $\mathrm{E} 2$

Stratégies affectives. Les stratégies affectives sont mises de l'avant pour comprendre ce que peuvent vivre certains élèves et les recentrer sur la tâche. Les 
enseignants font preuve de compréhension, de soutien et de compassion dans certains cas. Ils ont le souci de calmer les élèves, de les repositionner et ajustent parfois leurs interventions en fonction de la situation vécue par le jeune. Ils montrent une ouverture d'esprit au sujet de ce que peuvent vivre certains élèves tout en essayant de les ramener à la tâche en vue de la réussite du plus grand nombre d'entre eux.

Tristesse : Je lui suggère de mettre de côté la situation conflictuelle pour pouvoir être à l'écoute de la leçon. E2

Joie (surexcitation) : On va lui demander de se calmer, de se repositionner... le remettre en position pour que lui se questionne. E1

Désintérêt : Pourquoi tu fais ça? Pourquoi tu penses que c'est important de faire ça?... C'est vraiment de le ramener à la tâche... le non intérêt qui se manifeste à haute voix, la réponse va être à haute voix aussi. E1

Anxiété : Je vais ignorer la situation, puis revenir avec lui plus tard... reprendre en récupération. Il y a des moments où ça ne donne rien d'intervenir en présence de tous les élèves. E2

Somme toute, les stratégies d'intervention des enseignants mènent vers diverses actions. Les stratégies cognitives permettent de raisonner, de questionner et d'amener l'élève à s'autoréguler. Les stratégies kinesthésiques tendent à enlever, à replacer, à déplacer un élève. Les stratégies affectives pour certaines émotions (celles notées précédemment) amènent à discuter, à comprendre, à s'adapter, à faire prendre conscience et à accompagner.

\section{Discussion}

$\mathrm{Au}$ terme de cette recherche, nous pouvons confirmer que tous nos objectifs sont atteints. Cependant, il convient d'émettre quelques constatations. D'abord, les manifestations non verbales de l'écoute en classe sont rarement observées par les élèves et par les enseignants. Les postures démontrent un intérêt ou non pour la matière en cours. Certains gestes, volontaires ou non, aident à écouter, mais lorsqu'ils sont répétitifs et que le bruit y est associé, ils nuisent à l'écoute pour soi et pour les autres. Des situations émotionnelles particulières déconcentrent les élèves qui utilisent des stratégies pour écouter, mais qui, selon l'état du problème, retombent dans leurs préoccupations. Il convient de tenir compte de ces situations dans l'enseignement. Le contexte d'enseignement (approches pédagogiques utilisées) peut également influencer les stratégies d'écoute des élèves.

Ensuite, concernant les stratégies, les élèves comme les enseignants utilisent des stratégies kinesthésiques et cognitives. Ces stratégies sont centrées sur ce que les élèves font ou devraient faire pour écouter ou garder leur attention. Pour les élèves, les actions posées mettent à l'épreuve leur capacité d'écoute réelle, malgré le fait que l'activité peut leur sembler plus ou moins stimulante (Lafontaine et Pharand, 2011). Ces stratégies rejoignent dans une certaine mesure celles évoquées par Guérin (2008), comme sélectionner, répéter, décomposer, élaborer et organiser. Pour les enseignants, les rappels 
à l'ordre visent l'autodiscipline des élèves et le maintien de leur attention. Leurs stratégies d'intervention rejoignent notamment celles de Berthiaume et Daele (2010) comme le questionnement et la discussion. Par contre, aucune stratégie d'écoute n'est enseignée.

Les stratégies d'initiative des élèves ont pour but de comprendre la matière dans un climat de classe favorable relativement calme. Les stratégies affectives des enseignants dévoilent leur sensibilité et leur compréhension par rapport à des réalités moins agréables vécues par les élèves. Alors que des élèves disent essayer de ne pas penser à leurs problèmes en classe, d'autres ne peuvent s'empêcher d'exprimer certaines émotions que les enseignants remarquent. Les interventions de ces derniers semblent sporadiques et circonstancielles sans suivi annoncé. À l'instar de Bouchard (2003), les participants de cette recherche ont développé des stratégies pour surmonter certains obstacles, soit à leur enseignement ou à leurs apprentissages.

De plus, deux constats méritent d'être soulignés : 1) les postures, les gestes et les expressions faciales en tant qu'indices d'observation rendent possibles le repérage de certaines stratégies. Même si des stratégies ne s'observent pas facilement, l'enseignant doit se donner des moyens autres pour vérifier l'écoute; 2) le questionnement des enseignants est nécessaire pour s'assurer de la justesse des interprétations qu'ils font de ces indices. Sinon, il serait facile de présumer que tel élève n'écoute pas alors qu'il est capable de répondre à n'importe quelle question ou que tel autre écoute alors qu'il est dans ses pensées. Dans cette même optique, les élèves pourraient être sensibilisés au message contradictoire qu'envoient certaines de leurs stratégies d'écoute.

Dans cette perspective, il nous apparait important que les enseignants revoient leur interprétation de ce qu'est une manifestation positive de l'écoute des élèves. En effet, plusieurs stratégies évoquées par les élèves comme bailler, se coucher sur le pupitre, regarder dehors, jouer avec un objet sont des manifestations que les enseignants jugent négatives alors que pour les élèves, ces stratégies leur permettent de demeurer à l'écoute et de se concentrer.

La compréhension de l'oral passe par l'écoute. Le défi pour les élèves est de la maintenir et pour les enseignants, de la stimuler constamment. Ce qu'il faut retenir de cette étude, c'est l'importance de vérifier la correspondance entre les manifestations non verbales observées par l'enseignant et ce que vit ou ressent réellement l'élève. Pourquoi un tel a-t-il toujours besoin d'un objet à manipuler pour écouter? Tous les élèves ayant participé à cette recherche ont exprimé leur intention première d'écouter. C'est à partir de ce point de départ positif qu'il faut aborder l'écoute et observer les manifestations non verbales des élèves sachant qu'en cours de route, l'écoute se dilue pour une multitude de raisons auxquelles il faut s'intéresser pour garder le contact avec les élèves.

\section{Limites de la recherche}

Les résultats de notre recherche ne sont pas généralisables même s'ils dressent un portrait intéressant de deux classes de troisième cycle du primaire en ce qui concerne les manifestations non verbales et les stratégies d'écoute d'élèves ainsi que les stratégies d'intervention de leurs enseignants dans ce contexte. Une autre limite est l'approche pédagogique et les notions choisies lors de l'activité d'enseignement. Il va sans dire qu'il est moins intéressant pour un élève d'écouter un exposé magistral sur le participe passé que de voir son enseignant utiliser le tableau blanc numérique pour faire une animation 
en mathématiques ou de faire une activité d'enseignement coopératif par exemple. Il serait intéressant de reprendre nos outils lors de séances d'enseignement utilisant des approches pédagogiques plus interactives.

Une autre limite concerne le visionnage de la vidéo et le repérage des indices d'observation sur les fiches. Même si la caméra était déplacée pendant l'activité pour filmer tous les élèves et que la vidéo a été vue à deux reprises par chaque classe, il est possible que des indices aient échappés à l'œil des élèves. Cette limite ne les a toutefois pas empêchés de prendre conscience qu'ils font plusieurs gestes, qu'ils adoptent plusieurs postures et qu'ils expriment assez clairement leur intérêt ou non pour la matière. La dernière limite concerne l'analyse des réponses au questionnaire individuel. Pour la présente recherche, nous avons porté attention aux stratégies d'écoute notées, mais plusieurs autres réponses indiquent le malaise éprouvé par certaines élèves par rapport à des situations particulières vécues (encadré 2). Nous croyons qu'une analyse plus fine de ces autres réponses pourrait faire l'objet d'une publication ultérieure.

\section{Conclusion}

En conclusion, notre recherche a montré que des manifestations non verbales parfois connotées de façon négative font état d'une écoute des élèves. En effet, on entend souvent que pour écouter, il faut être assis bien droit, ne pas tenir d'objet, regarder la personne qui parle, etc. Les enseignants gagneraient-ils à ajuster, s'il y a lieu, leurs propres attitudes au sujet des postures d'écoute des élèves? Comme le dit cette élève : Je n'écoute pas toujours, mais ça ne paraît pas... je suis souvent dans la lune. E1-F4

L'écoute doit prendre une place plus importante dans la compétence à communiquer oralement, car sans écoute, intérêt et compréhension des messages, l'apprentissage et la réussite seront difficiles, particulièrement pour les élèves en difficulté (Dumais, 2010). Agir sur les manifestations non verbales de l'écoute observées permet aux élèves de réfléchir aux stratégies qu'ils adoptent pour garder leur attention ou se recentrer sur la tâche et aux enseignants d'utiliser des outils d'observation de l'écoute, d'intervenir et d'adapter leur enseignement en fonction de ces observations. Enfin, les stratégies d'écoute mériteraient d'être enseignées de manière explicite, notamment en formation initiale et continue des enseignants, car la compréhension de l'oral, transversale à toutes les disciplines d'enseignement, est une compétence à développer tout au long de la vie, Ainsi, l'enseignement de stratégies d'écoute et la prise de conscience de la part des élèves sont des pistes de recherche qui risquent d'être fécondes pour l'ensemble des matières à enseigner au primaire.

\section{Références}

Bardin, L. (2007). L'analyse de contenu, Paris, Presses Universitaires de France.

Bégin, C. (2008). Les stratégies d'apprentissage : un cadre de référence simplifié. Revue des sciences de l'éducation, 34, 47-67.

Berthiaume, D., \& Daele, A. (2010). Choisir ses stratégies d'enseignement. Suisse : Centre de soutien à l'enseignement, Université de Lausanne.

Bissonnette, S., Richard, M. \& Gauthier, C. (2005). Interventions pédagogiques efficaces et réussite scolaire des élèves provenant de milieux défavorisés. Revue française de pédagogie, 150, 87-141. 
Bouchard, G. (2003). Cognitive appraisals, neurism, and openness as correlates of coping strategies: an integrative model of adaptation to marital difficulties. Canadian Journal of Behavioural Science, 35, 1-12.

Christophe, V. (1998). Les émotions. Tour d'horizon des principales théories. Paris : Presses universitaires du Septentrion.

Dumais, C. (2010). Atelier pour un enseignement de 1'oral. Québec français, 157, 58-59.

Dolz, J., \& Schneuwly, B. (1998). Pour un enseignement de l'oral. Paris : ESF.

Lafontaine, L. (2007). Enseigner l'oral au secondaire. Séquences didactiques intégrées et outils d'évaluation. Montréal : Chenelière Éducation.

Lafontaine, L. (2008). Pratiques d'enseignement et d'évaluation de l'oral et perceptions d'enseignants de français et d'élèves du secondaire. Dans L. Lafontaine, R. Bergeron, \& G. Plessis-Bélair (dir.), L'oral dans les classes : pratiques innovantes (pp. 87-104). Québec : PUQ.

Lafontaine, L. (2013). Travailler la littératie volet oral au préscolaire et au 1er cycle du primaire : Ça nous parle! Vivre le primaire, 26, 1, p. 12-14.

Lafontaine, L., \& Pharand, J. (2011). Les stratégies d'écoute et leurs manifestations non verbales chez des élèves de $5^{\mathrm{e}}$ et $6^{\mathrm{e}}$ année. Vivre le primaire, $24(1), 45-48$.

Lafontaine, L., \& Le Cunff, C. (2005). Recherche comparée sur la prise de parole de certains élèves du préscolaire au primaire en français langue maternelle en France et au Québec. Éducation canadienne et internationale, 34(2), 72-97.

Lafontaine, L., \& Préfontaine, C. (2007). Modèle didactique descriptif de la production orale en classe de français langue première au secondaire. Revue des sciences de l'éducation, vol. XXXIII (1), 47-66.

Lafontaine, L., \& Messier, G. (2009). Les représentations de l'enseignement et de l'évaluation de l'oral chez des enseignants et des élèves du secondaire en français langue d'enseignement. Revue du Nouvel-Ontario, 34,119-144.

Laraoui, R., Morel, M., \& Leblanc, S. (2014). Des pratiques pédagogiques de l'enseignement du lire/écrire, déclarées par des enseignantes du primaire. Phronesis, 3 (1-2), 111-120.

Le Cunff, C. 2004. L’oral pour apprendre. Québec français, 133, 50-53.

Ministère de l'Éducation de l'Ontario (2006). Guide d'enseignement efficace en matière de littératie de la $4^{e}$ à la $6^{e}$ année Fascicule 1 : Grandir avec la littératie. Toronto : Imprimeur de la Reine.

Ministère de l'Éducation de l'Ontario (2008). Guide d'enseignement efficace de la communication orale de la maternelle à la $3^{e}$ année. Gouvernement de l'Ontario.

Ministère de l'éducation du Québec (2001). Programme de formation de l'école québécoise. Éducation préscolaire et enseignement primaire. Québec : Gouvernement du Québec.

Ministère de l'éducation, du loisir et du sport (2011). Progression des apprentissages au primaire. Français, langue d'enseignement, en ligne.www1.mels.gouv.qc.ca/sections/programme

Mordaunt, O.G., \& Olson, D.W. (2010). Listen, listen, listen and listen: building a comprehension corpus and making it comprehensible. Educational Studies, 36 (3), 249-258. 
Nolin, R. (2013). Pratiques déclarées d'enseignement et d'évaluation de l'oral d'enseignants du primaire au Québec. Mémoire de maitrise inédit, Université du Québec à Montréal.

Plessis-Bélair, G. 2004. La communication orale. Québec français, 133, 57-59.

Pharand, J. (2013). Des manifestations à la compréhension des émotions en contexte d'enseignement au primaire. Dans J. Pharand, et M. Doucet (Dir.), En éducation, quand les émotions s'en mêlent. Enseignement, apprentissage et accompagnement (pp. 35-60). Québec : PUQ.

Pharand, J. (2005). La part des émotions dans la communication entre l'enseignant et les élèves du primaire lors de séquences d'enseignement [CD-ROM]. Dans les Actes du $5^{\mathrm{e}}$ Colloque International Recherche(s) et Formation, Former des enseignantsprofessionnels, savoirs et compétences. Nantes : IUFM des Pays de la Loire et Centre de Recherche en Éducation de l'Université de Nantes.

Savoie-Zajc, L. (2000). La recherche qualitative/interprétative en éducation. Dans T. Karsenti, \& L. Savoie-Zajc (dir.), Introduction à la recherche en éducation (p. 172198). Sherbrooke : Éditions CRP.

Watzlawick, P., Helmick-Beavin, J., \& Jackson, D.D. (1972). Une logique de la communication. Paris : Éditions du Seuil.

\section{Les biographies des auteurs}

Joanne Pharand est professeure agrégée en formation pratique à l'Université du Québec en Outaouais, campus de Saint-Jérôme, où elle est responsable pédagogique des stages au baccalauréat en éducation préscolaire et enseignement primaire et responsable de la formation des enseignants associés. Elle est membre d'ÉRLI (Équipe de recherche en littératie et inclusion) et s'intéresse particulièrement à la littératie émotionnelle au primaire en enseignement/apprentissage. Ses recherches portent aussi sur les compétences attendues des principaux acteurs impliqués dans les stages ainsi que sur l'autoformation en insertion professionnelle.

Lizanne Lafontaine est professeure titulaire de didactique du français au Département des sciences de l'éducation de l'Université du Québec en Outaouais, campus SaintJérôme, et responsable de l'Équipe de recherche en littératie et inclusion (ÉRLI) de la même université. Ses recherches portent sur la didactique de l'oral au préscolaire, au primaire et au secondaire ainsi que sur la littératie en milieu défavorisé. 